\title{
Effect of meat product with onion skin extract on metabolic profile in SHR
}

\section{Tetyana Ivanova ${ }^{1}$, Yulia Goshovska ${ }^{2}$, Lyudmyla Peshuk ${ }^{1}$, Mariana Romanenko ${ }^{3}$, Raisa Fedichkina ${ }^{2}$, Vadym Sagach ${ }^{2}$, Lyudmyla Sineok ${ }^{3}$, Igor Shapoval ${ }^{3}$}

1 - National University of Food Technologies, Kyiv

2 - Bogomoletz Institute of Physiology of National Academy of Science of Ukraine, Kyiv 3 - Chebotarev State Institute of Gerontology of National Academy of Medical Science of Ukraine, Kyiv

\section{Keywords:}

Onion

Extract

Glucose

Lipids

Rat

\section{Article history:}

Received 26.12.2016

Received in revised form 19.02.2017

Accepted 30.03.2017

Corresponding author:

Tetyana Ivanova

E-mail:

Tatiana_n.iv@ukr.net

DOI:

$10.24263 / 2304-$

974X-2017-6-1-10

\section{Abstract}

Introduction. Searching for the new product compositions is important for metabolic disorders correction. Onion contains flavonoid quercetin with antioxidant and cardioprotective effect.

Materials and methods. Meat product with an onion skin extract or quercetin at equal dose $2,25 \mathrm{mg}$ of quercetin daily was used for animals feeding. Six-month-old spontaneous hypertensive rats (SHR) with fructose overload in drinking water $(25 \%)$ were used to promote metabolic disorders. After 3 months of feeding glucose was measured in arterial blood by glucose sensors based on amperometric measurement. Plasma lipid profile was determined by an enzymatic colorimetric reaction.

Results and discussion. Blood glucose in SHR without diet interventions (group II) was $59 \%$ higher than in control $(8.84 \pm 0.3$ $\mathrm{mmol} / \mathrm{l}$ vs $5.56 \pm 0.86 \mathrm{mmol} / \mathrm{l}, \mathrm{P}<0.01)$. Fructose overload in SHR (group III) increased glucose level on $5 \%(9.3 \pm 0.4 \mathrm{mmol} / \mathrm{l}, \mathrm{P}>0.05$ compared to SHR of group II drinking tap water). Quercetin added to the meat product did not influence glucose concentration in SHR on fructose overload (group IV) $-9.2 \pm 0.8 \mathrm{mmol} / 1, \mathrm{P}>0.05$ compared to SHR of groups II and III. In SHR on fructose overload receiving meat product with onion skin extract (group V) blood glucose tended to decrease $-8.0 \pm 1.0 \mathrm{mmol} / \mathrm{l}, \mathrm{P}>0.05$ compared to SHR of group III.

Total cholesterol tended to increase in SHR of group II compared to control $(1.36 \pm 0.10$ vs $1.22 \pm 0.05 \mathrm{mmol} / \mathrm{l}, \mathrm{P}>0.05)$. In SHR of group III was significantly higher than in control $(1.45 \pm 0.09 \mathrm{mmol} / \mathrm{l}$, $\mathrm{P}=0.03)$ but did not differ from SHR drinking tap water $(1.45 \pm 0.09$ vs $1.36 \pm 0.10, \mathrm{P}>0.05)$. In groups IV and $\mathrm{V}$ total cholesterol was also significantly higher than in control $(1.52 \pm 0.07$ and $1.57 \pm 0.09$ vs $1.22 \pm 0.05 \mathrm{mmol} / \mathrm{l}, \mathrm{P}=0.006$ and $\mathrm{P}=0.005$, respectively).

The rise of total cholesterol observed in the SHR of groups IV and $\mathrm{V}$ was due to the increase of high-density lipoprotein (HDL) cholesterol because non-HDL-cholesterol did not change in them. In SHR supplemented by meat product with onion skin extract there was a significant increase in HDL-cholesterol compared to SHR of group II ( $1.25 \pm 0.09$ vs $1.02 \pm 0.05, \mathrm{P}=0.03)$. An increase in HDL cholesterol was also observed in SHR receiving meat product containing purified quercetin powder compared to SHR of group II $(1.23 \pm 0.08$ vs $1.02 \pm 0.05, \mathrm{P}=0.02)$.

Conclusions. Supplementation with the meat product containing onion skin extract to SHR with fructose overload has a positive effect on blood glucose. The increase of HDL-cholesterol in SHR with fructose overload reflects anti-atherogenic effect of the developed meat product. 


\section{Introduction}

Nowadays cardiovascular diseases (CVD) remains the important problem for health care service all over the world. CVD are the leading cause of death globally, most occurred in low and middle income countries [1]. High prevalence of CVD is supported by rising amount of people with metabolic syndrome, consisting of obesity, arterial hypertension, lipid and glucose disorders. According to WHO, CVD prevalence can be decreased by the influence on diet and body weight among others factors [1]. So nutrition researches and food industry need to search for the new product compositions to prevent and correct metabolic disorders.

The most important and widely cultivated vegetable

is onion (Allium cepa L.). The onion world manufacture is about 55 billion ton per year (Tina et al., 2016; Radga et al., 2016). This vegetable contains flavonoids with antioxidant and cardioprotective effect. Of particular interest is yellow onion - the most spread cultured onion in Ukraine.

At that, yellow onion contains the highest total amount of flavonoids among the other onion varieties tested [2]. According to the researchers, flavonoids of this culture have high content of quercetin $(40000 \mathrm{mg} / \mathrm{kg})[3]$.

Quercetin $\left(3,3^{\prime}, 4^{\prime}, 5,7\right.$-pentahydroxyflavone) is a potent antioxidant, known for his antiinflammatory activity. This flavonoid possess wide range of positive effects, including neuroprotective, cardioprotective and even life-span extending effects [4]. Quercetin protects blood vessels from the damaging effects of oxidative stress, especially from lipid peroxidation, decreases blood pressure, leads to lowering of LDL cholesterol and causes cardioprotective effect. Results from human randomized controlled trials showed that flavonol consumption, especially quercetin, improved biomarkers of CVD risk [5]. Dietary supplementation with quercetin significantly reduced systolic blood pressure in overweight subjects with a high CVD risk and decreased plasma oxidised LDL concentrations that reflects cardioprotective effect of flavonoid [6]. According to meta-analyses quercetin supplementation was associated with a positive effect on blood lipid profile. It was observed in small but significant decrease in total cholesterol, LDL cholesterol and triacylglycerol and significant increase in HDL cholesterol [5].

Animal studies have demonstrated as the anti-inflammatory effect of quercetin as its possibility to modify blood lipid profile [7-9]. Quercetin was able to reduce liver injury in the mice model of diet-induced steatohepatitis. Noteworthy, steatosis and steatohepatitis is common liver injury in patients with metabolic syndrome. Given orally at dose $50 \mathrm{mg} / \mathrm{kg}$ daily quercetin markedly suppressed lipoperoxidation in liver tissue, decreased profibrotic and proinflammatory gene pathways in mice with steatohepatitis compared to control group. According to investigators, attenuation of proinflammatory and profibrotic pathways was due to anti-inflammatory effect of flavonoid [7]. It is known that liver function plays the key role in lipid homeostasis. Supplementation with quercetin to a commercial chow $(0.08 \%$ of the diet) influenced lipid profile in leptin receptor-deficient $\mathrm{db} / \mathrm{db}$ mice, which has metabolic disorders associated with metabolic syndrome and type 2 diabetes. In this experiment quercetin decreased plasma total cholesterol and increased HDL-cholesterol compared with the control [8].

Different animal models are known for studying metabolic syndrome. One of them spontaneously hypertensive rats (SHR) with genetic predisposition to high blood pressure and insulin resistance. This rat strain was chosen because arterial hypertension is prevalent CVD in Ukraine and is one of key components of metabolic syndrome. To provoke metabolic disorders in animals carbohydrate or fat overload is usually used [10-12]. We 
choose carbohydrate overload, according to modern nutritional pattern of Ukrainian people with metabolic syndrome. The aim of our studying was to investigate the metabolic profile in SHR kept at high-fructose diet under the supplementation with meat product containing onion skin extract, rich source of quercetin.

\section{Materials and methods}

\section{Investigated materials}

A meat product with onion skin extract, a meat product with purified quercetin powder, six-month-old rats with genetically determined hypertension under fructose overload, blood glucose and lipid profile.

\section{Description of the methods and facilities}

Preparation of onion skin extract for meat product. For animal feeding meat product with onion skin extract was used. The onion skin extract for the meat product was prepared in this way: electro-activated water was bringing to its boiling, weighted onion peel was added to boiling water in amount of $4 \%$ and was boiled for $10 \mathrm{~min}$ and filtered. Earlier the prepared onion skin extract was investigated and its quercetin content was determined [18].

The obtained extract was evaporated to 10 times smaller volume and was added to the meat product. Experimental animals received 1 gram of meat product with added 1,5 $\mathrm{ml}$ of the onion skin extract containing $2,25 \mathrm{mg}(0,00225 \mathrm{~g})$ of quercetin per rat daily during three months. In the prepared meat product the quercetin content was equal to $0,2 \%$ (or $7,5 \mathrm{mg}$ of quercetin per $\mathrm{kg}$ of rat body mass). Feeding these products to rats was performed orally.

Animals and experimental diet. For studying quercetin influence on metabolic profile we used male SHR. This rat strain reveals genetic predisposition to metabolic disorders and is one of proposed animal model for metabolic syndrome among others. As control were taken male Wistar rats. Six-month-old rats were included in experiment. Animals were housed at vivarium standard conditions $5-6$ rats in a cage at $22-24^{\circ} \mathrm{C}$ and maintained on a standard laboratory rat chow ad libitum.

Fructose overload in drinking water was used to promote metabolic disorders. Fructose overload in experimental animals is associated with increased body mass, insulin resistance, glucose and lipid metabolism disorders [10, 13-14]. High-fructose diet is also used in SHR to develop metabolic syndrome [10, 15].

Rats were divided into 4 groups (5-6 rats per group). The first, control group (I) Wistar rats kept on the standard laboratory chow plus drinking tap water. The second (II) SHR kept on standard laboratory chow plus drinking tap water. The third (III) - SHR kept on standard laboratory chow plus $25 \%$ fructose solution in drinking water [13]. The fourth (IV) - SHR received meat product with pure 96\% quercetin powder added (Merk, Germany) and 25\% fructose solution in drinking water. The fifth (V) - SHR received meat product with onion skin extract added and $25 \%$ fructose solution in drinking water. All series lasted for 3 months.

Sampling procedures. Animals were sacrificing by decapitation at the end of the treatment after an overnight fasting and blood samples were immediately collected. Blood 
samples were centrifuged at $1,500 \mathrm{~g}$ for $15 \mathrm{~min}$ to acquire plasma. Plasma samples were stored at $-70^{\circ} \mathrm{C}$ for further analysis.

Blood glucose. After sacrificing the rats glucose was measured in arterial blood by using glucose sensors which worked on the basis of amperometric measurement after enzymatic glucose oxidation (Accu-Chek Active, Roche, Germany) [16].

Lipid concentration in the plasma. Plasma triglycerides, total cholesterol, HDLcholesterol level were estimated using a commercially available test kit (Biosystems, Spain) with an automated analyzer of an enzymatic colorimetric reaction Avtolab 18 (Mannhein Boehringer, Germany) [17].

For total cholesterol measurement reagents and plasma were distributed on labeled test tubes, mixed thoroughly and incubated for 5 minutes at $37{ }^{\circ} \mathrm{C}$. The absorbance of the standard and sample were measured by automated analyzer at $500 \mathrm{~nm}$ against the blank. After that total cholesterol concentration was calculated.

For triglycerides measurement reagents and plasma were distributed on labeled test tubes. The content of the tubes was thoroughly mixed and incubated at $37^{\circ} \mathrm{C}$ for 5 minutes. The absorbance of the standard and sample were measured by automated analyzer at 500 $\mathrm{nm}$ against the blank and triglycerides concentration was calculated.

For HDL cholesterol measurement reagents and plasma were distributed on centrifuge tubes, mixed thoroughly and left for 10 minutes. After that tubes were centrifuged at 4000 r.p.m. for 10 minutes. The supernatant was collected. The reagent, sample supernatant, standard and distilled water were distributed on labeled test tubes, mixed thoroughly and incubated for 10 minutes at $37{ }^{\circ} \mathrm{C}$. The absorbance of the standard and sample were measured by automated analyzer at $500 \mathrm{~nm}$ against the blank. After that HDL cholesterol concentration was calculated using sample dilution factor.

Non-HDL cholesterol was calculated by formula: non-HDL cholesterol $=$ total cholesterol - HDL cholesterol.

Statistical analysis. Data presented as means and standard errors of the means. The analysis was performed with the use of Statistica 6.0 software. The t-test for unpaired samples was used for group comparisons. The results were considered significant with a $\mathrm{P}$ value of less than 0.05 .

\section{Results and discussion}

Arterial blood glucose in SHR of group II was $59 \%$ higher than in control $(8.84 \pm 0.3$ $\mathrm{mmol} / \mathrm{l}$ versus $5.56 \pm 0.86 \mathrm{mmol} / \mathrm{l}, \mathrm{P}<0.01$; Figure 1$)$. Fructose overload in drinking water increased glucose level on $5 \%$ in group III $(9.3 \pm 0.4 \mathrm{mmol} / 1, \mathrm{P}>0.05$ compared to group II). Quercetin added to the meat product (group IV) did not influence glucose concentration in rats with fructose overload $-9.2 \pm 0.8 \mathrm{mmol} / 1, \mathrm{P}>0.05$ compared to group II and group III. Blood glucose in group $\mathrm{V}$ at the onion skin extract was $8.0 \pm 1.0 \mathrm{mmol} / 1, \mathrm{P}>0.05$ compared to groups II - IV.

However supplementation with the meat product containing onion skin extract to rats tended to decrease glucose level - in group V it was 14\% lower compared to SHR of group III, receiving standard chow and $25 \%$ fructose solution in drinking water. This corresponds 
to study by V. Brüll et al., who found quercetin from onion skin extract did not decrease glucose level in subjects with metabolic syndrome and hypertension [20].

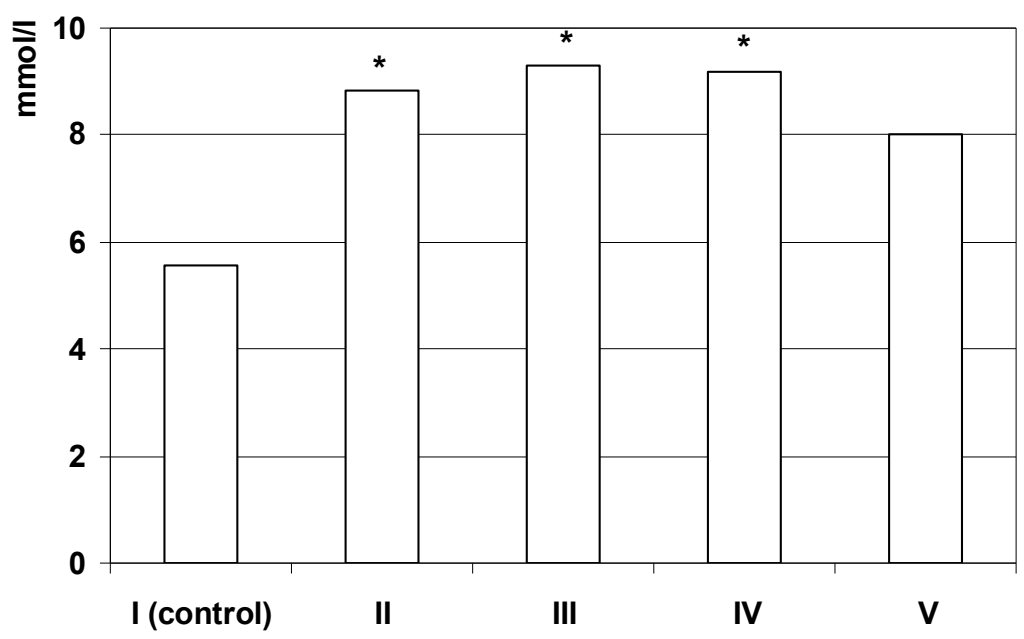

Group number

Figure 1. Blood glucose level in rats with the different diet interventions * - significant difference versus control, $\mathbf{P}<0.05$

On the other hand, fructose overload as $10 \%$ solution in drinking water for two months in Wistar rats caused the rise of blood glucose [14]. SHR fed with commercial chocolate bars for 12 weeks also revealed elevated glucose level compared to standard chow. These rats have increased body weight due to caloric exceed. Also they showed higher insulin and C-peptide level [12]. It is noteworthy that we had initially high glucose in the rats with genetically determined hypertension. In these conditions dietary intervention with fructose and the meat product may do not cause obvious changes in glucose metabolism. So we did not receive an additional significant rise of blood glucose over initial level under the fructose overload.

Comparing two rat strains, M. Oron-Herman et al. showed that sucrose supplementation for 7 weeks failed to rise markedly glucose level in SHR. Authors noted that SHR strain has initially impaired glucose tolerance and represents metabolic syndrome. So it is hard to cause in them metabolic disorders progression to diet interventions compared to other rat strain without hypertension. However sucrose overload caused further elevation of blood pressure in SHR [11].

Weight gain in all groups did not differ during and at the end the study. This corresponds to study by M. Oron-Herman et al., in which sucrose supplementation failed to cause an obesity [11]. A. Zemančíková and J. Török also did not find an increase in body weight of Wistar rats drinking $10 \%$ fructose solution for 8 weeks in spite of plasma glucose rise [14].

Results of total cholesterol, triglycerides, HDL-cholesterol and non-HDL-cholesterol are presented in the Table 1 . 
Lipid profile in rats with the different diet interventions

\begin{tabular}{|l|c|c|c|c|c|}
\hline & \multicolumn{1}{|c|}{$\begin{array}{c}\text { I } \\
\text { Control } \\
\mathbf{n = 6}\end{array}$} & $\begin{array}{c}\text { II } \\
\text { SHR } \\
\mathbf{n = 5}\end{array}$ & $\begin{array}{c}\text { III } \\
\text { SHR }+ \\
\text { fructose } \\
\text { overload } \\
\mathbf{n = 6}\end{array}$ & $\begin{array}{c}\text { IV } \\
\text { SHR + } \\
\text { fructose } \\
\text { overload } \\
\text { quercetin } \\
\mathbf{n = 5}\end{array}$ & $\begin{array}{c}\text { SHR + fructose } \\
\text { overload } \\
+ \text { onion skin } \\
\text { extract } \\
\mathbf{n}=\mathbf{6}\end{array}$ \\
\hline $\begin{array}{l}\text { Total cholesterol, } \\
\text { mmol/1 }\end{array}$ & $1.22 \pm 0.05$ & $1.36 \pm 0.10$ & $1.45 \pm 0.09 *$ & $1.52 \pm 0.07 *$ & $1.57 \pm 0.09 *$ \\
\hline $\begin{array}{l}\text { Triglycerides, } \\
\text { mmol/1 }\end{array}$ & $0.64 \pm 0.03$ & $0.64 \pm 0.06$ & $0.65 \pm 0.05$ & $0.66 \pm 0.10$ & $0.65 \pm 0.07$ \\
\hline $\begin{array}{l}\text { HDL-cholesterol, } \\
\text { mmol/1 }\end{array}$ & $0.93 \pm 0.03$ & $1.02 \pm 0.05$ & $1.09 \pm 0.05^{*}$ & $1.23 \pm 0.08^{*} \&$ & $1.25 \pm 0.09 * \&$ \\
\hline $\begin{array}{l}\text { Non-HDL- } \\
\text { cholesterol, } \\
\text { mmol/1 }\end{array}$ & $0.29 \pm 0.05$ & $0.34 \pm 0.05$ & $0.36 \pm 0.10$ & $0.29 \pm 0.03$ & $0.38 \pm 0.05$ \\
\hline
\end{tabular}

* - significant difference versus control, $\mathrm{P}<0.05$;

\& - significant difference versus SHR (II group).

Total cholesterol tended to increase in SHR of group II compared to control $(\mathrm{P}>0.05)$. In SHR with fructose overload it was also significantly higher than in control Wistar rats $(\mathrm{P}=0.03)$ but do not differ from SHR drinking tap water. Our results are in accordance with study by M. Oron-Herman et al. Earlier they found slightly elevated total cholesterol in SHR fed either standard laboratory chow or sucrose without any difference between groups [11]. In groups IV and V total cholesterol was also significantly higher than in control ( $\mathrm{P}=0.006$ and $\mathrm{P}=0.005$, respectively). The rise of total cholesterol observed in the different SHR groups was due to the increase of HDL-cholesterol because non-HDL-cholesterol did not change in them. HDL-cholesterol was increased in all groups with fructose overload compared to control Wistar rats. In SHR on fructose overload and receiving meat product with quercetin HDL-cholesterol was greater elevated compared to SHR drinking tap water $(\mathrm{P}=0.02)$. The same increase was observed for group receiving the meat product with onion skin extract compared to SHR of group II $(\mathrm{P}=0.03)$. Noteworthy, the SHR strain has a genetic background of insulin resistance which usually is characterized by low HDLcholestrol [20]. The rise of HDL-cholesterol is positive phenomenon because of its antiatherogenic effect. In this case we can see similar effect of purified quercetin powder and onion skin extract added to the meat product.

Triglycerides did not differ in all experimental groups and control. M. Oron-Herman et al. also showed that triglycerides did not change in SHR under sucrose supplementation [11]. In contrary, J. Török et al. received increased triglycerides in Wistar rats and SHR drinking water containing $10 \%$ fructose for two month [15].

In above mentioned animal studies of carbohydrates overload for developing of metabolic syndrome different lipid profile results can be in part explained by rat strain, type of carbohydrates used and experiment design [12].

Literature data conserning the quercetin effect on lipid profile are a little bit contradictory. In human studies quercetin supplementation did not influence lipid profile in the subjects with metabolic syndrome and pre-hypertension or stage I hypertension despite 
of lowering of blood pressure in patients with hypertension. Authors did not find significant differences between quercetin supplemented group and placebo group in serum total cholesterol, LDL-cholesterol, HDL-cholesterol and tryacilglycerol levels [19]. In study by S. Egert et al. the decrease of oxidized LDL-cholesterol consentration was shown, but serum HDL-cholesterol and LDL-cholesterol levels remained unchanged [6]. Consistent with the our findings, increased HDL-cholesterol was also reported by K.H. Lee et al. in healthy male smokers supplemented with quercetine from onion peel [21].

Thus, our data indicate that dietary intervention with the onion skin extract rich in quercetin at dose $7,5 \mathrm{mg} / \mathrm{kg}$ may have positive influence on glucose and lipid profile.

Furthermore, our data showed non toxic effect of suggested meet supplement with onion skin extract that might be introduced for the development of new food product with hypoglycemic properties and modulating lipid metabolism. Further studies are needed to investigate the effect of supplementation with the meat product containing onion skin extract on more wide range of metabolic risk factors.

\section{Conclusions}

1. Supplementation with the meat product containing onion skin extract to SHR at dose $2,25 \mathrm{mg}(0,00225 \mathrm{~g})$ of quercetin daily is safe.

2. Supplementation with the meat product containing onion skin extract tended to decrease glucose level in SHR on fructose overload

3. There was a positive effect of the meat product containing either purified quercetin powder or onion skin extract on HDL-cholesterol in SHR with fructose overload. Rise of HDL-cholesterol reflects better anti-atherogenic effect of the developed meat product.

\section{References}

1. Roth G.A., Johnson C., Abajobir A. et al. (2017), Global, Regional, and National Burden of Cardiovascular Diseases for 10 Causes, Journal of the American College of Cardiology, 17, p. 2687.

2. Yang J., Meyers K.J., Van Der Heide J., Liu R.H. (2004), Varietal differences in phenolic content and antioxidant and antiproliferative activities of onions, Journal of Agricultural and Food Chemistry, 52(22), pp. 6787-6793.

3. Lakhanpal P., Deepak Kumar Rai. (2007), Quercetin: a versatile flavonoid, IJMU, 2(2), pp. 24-28.

4. Ed. By Vaiserman A.M. (2017), Anti-aging drugs: From basic research to clenical practice, Royal Society of Chemistry, p. 570.

5. Menezes R., Rodriguez-Mateos A., Kaltsatou A. (2017), Impact of flavonols on cardiometabolic biomarkers: a meta-analysis of randomized controlled human trials to explore the role of inter-individual variability, Nutrients, 9(2), p. 117.

6. Egert S., Bosy-Weestphal A., Seiberl J. (2009), Quercetin reduces systolic blood pressure and plasma oxidised low density lipoprotein concentrations in overweight subjects with a high-cardiovascular disease risk phenotype: a double-blinded, placebocontrolled cross-over study, Br J Nutr, 102, pp. 1065-1074. 
7. Marcolin E., San-Miguel B., Vallejo D. (2012), Quercetin treatment ameliorates inflammation and fibrosis in mice with nonalcoholic steatohepatitis, The Journal of Nutrition, pp. 1-8.

8. Jeong S.M, Kang M.J, Choi H.N. (2012), Quercetin ameliorates hyperglycemia and dyslipidemia and improves antioxidant status in type 2 diabetic $\mathrm{db} / \mathrm{db}$ mice, Nutr Res Pract, 6, pp. 201-207.

9. Gnoni G.V., Paglialonga G., Siculella L. (2009), Quercetin inhibits fatty acid and triacylglycerol synthesis in rat-liver cells, Eur J Clin Invest, 39, pp.761-768.

10. Lehnen A.M., Rodrigues B., Irigoyen M.C. (2013), Cardiovascular Changes in Animal Models of Metabolic Syndrome, J. Diabetes Res, 76, pp. 13-14.

11. Oron-Herman M., Kamari Y., Grossman E. et al. (2008), Metabolic syndrome: comparison of the two commonly used animal models, Am J Hypertens, pp. 10181022 .

12. Miesel A., Müller H., Thermann M. et al. (2010), Overfeeding-induced obesity in spontaneously hypertensive rats: an animal model of the human metabolic syndrome, Ann Nutr Metab, 56(2), pp. 127-142.

13. Levi B., Werman M.J. (1998), Long-Term Fructose Consumption Accelerates Glycation and Several Age-Related Variables in Male Rats, J. Nutr. 128(9), pp. 14421449.

14. Zemančíková A., Török J. (2014), Cardiovascular effects of high-fructose intake in rats with nitric oxide deficiency, Interdisci.p Toxicol, 7(3), pp. 159-164.

15. Torok J., Zemancikova A., Tabacekova M. (2012), Effect of high-fructose intake on cardiovascular function in normotensive and hypertensive rats, Acta Physiologica, 206(693), p. 86.

16. Gan K.X., Wang C., Chen J.H. et al. (2013), Mitofusin-2 ameliorates high-fat dietinduced insulin resistance in liver of rats, World J Gastroenterol, 19(10), pp. 15721581.

17. Burtis C.A., Ashwood E.R., Bruns D.E. (2006), Tietz Textbook of Clinical Chemistry and Molecular Diagnostics, 4th ed., St. Louis, MO: Elsevier Saunders, p. 2448.

18. Peshuk L.V., Havalko Y.V., Ivanova T.N. (2016), Prospects for the use of secondary raw materials kvertsetynvmisnoyi (peel onion and garlic) herbs and special technology in meat products, Scientific Work of National University of Food Technologies, 22(5), pp. 238-244.

19. Brüll V., Burak C., Stoffel-Wagner B. (2015), Effects of a quercetin-rich onion skin extract on $24 \mathrm{~h}$ ambulatory blood pressure and endothelial function in overweight-toobese patients with (pre-)hypertension: a randomised double-blinded placebo-controlled cross-over trial, Br J Nutr, 114(8), pp. 1263-1277.

20. Pravenec M., Zídek V., Musilová A. et al. (2002), Incorporating Mouse Genome, Mammalian Genome,13, pp. 253-258.

21. Lee K.-H., Park E., Lee H-J et al. (2011), Effects of daily quercetin-rich supplementation on cardiometabolic risks in male smokers, Nutrition Research and Practice, 5(1), pp. 28-33. 